\title{
Collision case model for population balance equations in agglomerating heterogeneous colloidal systems: Theory and experiment
}

\author{
Frank Rhein*, Felix Ruß, Hermann Nirschl \\ Karlsruhe Institute of Technology (KIT), Institute for Mechanical Process Engineering and Mechanics, Strasse am Forum 8, 76131 Karlsruhe, Germany
}

G R A P H I C A L A B S T R A C T

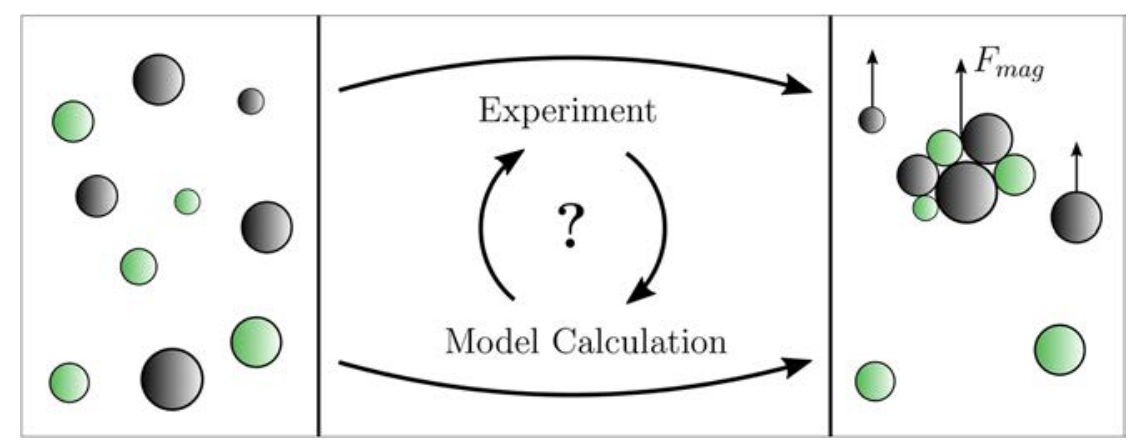

Keywords:

Magnetic seeded filtration

Population balances

Hetero Agglomeration
A B S T R A C T

The main goal of this work is to develop and validate a new method for characterizing the material properties of hetero-agglomerates when calculating population balances in magnetic seeded filtration processes. A new approach of computing various collision cases and probabilities and then weighting the respective agglomeration rates with them is considered to be physically justifiable. An experimental design of experiment (DOE) yielded data for the variation of multiple process parameters with a reasonable standard deviation. By measuring agglomeration kinetics, a deeper understanding of the process could be achieved and simple mathematical models were found in order to describe experimental data. The following multiple regression analysis showed good accordance with the agglomeration theory and thus the data was usable for further validation of the calculation results. By comparing calculated and experimental data and minimizing the residuals, estimates of the model parameters describing hard-to-measure material properties could be obtained. The comparison of experimental and computational regression models showed good agreement and thus validates the fundamental physical soundness of the implemented model. Furthermore, the comparison of the measured and calculated agglomeration kinetics further suggests the validity of the collision case model. Finally, this work showcases the possibilities of additional model calculations for a deeper, more thorough process understanding.

\section{Introduction}

Developments in separation technology have only been able to keep pace with advances in particle production to a limited extent. The current separation equipment is often optimized for processes on the basis of a single or two material features. Centrifuges are, for example, mainly based on density and particle size, whilst chromatographic methods can be used to separate the different types of particles on the

\footnotetext{
* Corresponding author.

E-mail address: frank.rhein@kit.edu (F. Rhein).
} 


\begin{tabular}{|c|c|c|c|}
\hline \multicolumn{2}{|c|}{ Nomenclature } & $r_{i, j}$ & radius of particle $A_{i, j}[\mathrm{~m}]$ \\
\hline$A_{H}$ & HAMAKER CONSTANT $[\mathrm{J}]$ & $T$ & temperature $[\mathrm{K}]$ \\
\hline$A_{\lambda}$ & absorbance at wavelength $\lambda\left[\mathrm{cm}^{-1}\right]$ & $T_{N M}$ & separation efficiency for the non-magnetic particles [\%] \\
\hline$C_{1}$ & $\begin{array}{l}\text { strength parameter short-ranged hydrophobic interaction } \\
{\left[\mathrm{Nm}^{-1}\right]}\end{array}$ & $\begin{array}{l}\Delta t \\
t_{\mathrm{A}}\end{array}$ & $\begin{array}{l}\text { time step size }[\mathrm{s}] \\
\text { agglomeration time }[\mathrm{s}]\end{array}$ \\
\hline$C_{2}$ & $\begin{array}{l}\text { strength parameter long-ranged hydrophobic interaction } \\
{\left[\mathrm{Nm}^{-1}\right]}\end{array}$ & $\begin{array}{l}V_{S} \\
x_{50}\end{array}$ & $\begin{array}{l}\text { volume of suspension }\left[\mathrm{m}^{3}\right] \\
\text { mean diameter }[\mathrm{m}]\end{array}$ \\
\hline$c_{v, M}$ & volume concentration magnetic particles [vol\%] & $Y_{N M}$ & non-magnetic surface fraction $[-]$ \\
\hline$c_{v, \mathrm{NM}}$ & volume concentration non-magnetic particles [vol\%] & $\alpha_{i j, l m}$ & collision efficiency between $A_{i, j}$ and $A_{l, m}\left[\mathrm{~m}^{-3}\right]$ \\
\hline$d_{1}$ & decay length short-ranged hydrophobic interaction $[\mathrm{m}]$ & $\beta_{i j, l m}$ & collision frequency between $A_{i, j}$ and $A_{l, m}\left[\mathrm{~m}^{3} \mathrm{~s}^{-1}\right]$ \\
\hline$d_{2}$ & decay length long-ranged hydrophobic interaction [m] & $\chi$ & susceptibility $[-]$ \\
\hline$e$ & electron charge $[\mathrm{C}]$ & $\kappa$ & reciprocal DEBYE length $\left[\mathrm{m}^{-1}\right]$ \\
\hline$F_{i j, l m}$ & agglomeration efficiency between $A_{i, j}$ and $A_{l, m}\left[\mathrm{~s}^{-1}\right]$ & $\lambda$ & wavelength $[\mathrm{m}]$ \\
\hline G & mean shear rate $\left[\mathrm{s}^{-1}\right]$ & $\mu$ & dynamic viscosity [Pa s] \\
\hline$h$ & absolute surface-to-surface distance $[\mathrm{m}]$ & $\Phi_{v, \mathrm{NM}}$ & volume concentration ratio non-magnetic particles \\
\hline$I$ & ionic strength $\left[\frac{\mathrm{mol}}{\mathrm{m}^{3}}\right]$ & & $\Phi_{v, \mathrm{NM}}=\frac{c_{v, \mathrm{NM}}}{c_{v, M}}[-]$ \\
\hline$k$ & Boltzmann constant $\left[\mathrm{JK}^{-1}\right]$ & $\varphi$ & surface potential $[\mathrm{V}]$ \\
\hline$M$ & magnetization $\left[\frac{\mathrm{A}}{\mathrm{m}}\right]$ & $\Psi_{\Sigma}$ & sum of interaction potentials $[\mathrm{J}]$ \\
\hline$N$ & number concentration matrix $[-]$ & $\Psi_{e l}$ & electrostatic interaction potential $[\mathrm{J}]$ \\
\hline & AVOGADRO NUMBER $\left[\mathrm{MOL}^{-1}\right]$ & $\Psi_{h p h}$ & hydrophobic interaction potential [J] \\
\hline$N_{S, \max }$ & grid parameter, maximum number of primary particles per & $\Psi_{\nu d W}$ & Van-der-Waals interaction potential $[\mathrm{J}]$ \\
\hline$n$ & $\begin{array}{l}\text { agglomerate }[-] \\
\text { rotary speed }\left[\mathrm{s}^{-1}\right]\end{array}$ & $\begin{array}{l}\rho \\
\Theta\end{array}$ & $\begin{array}{l}\text { density }\left[\frac{\mathrm{kg}}{\mathrm{m}}\right] \\
\text { contact angle }\left[^{\circ}\right]\end{array}$ \\
\hline$P$ & probability $[-]$ & $\zeta$ & zeta potential $[\mathrm{V}]$ \\
\hline $\mathrm{Pe}$ & PÉCLET NUMBER [-] & & \\
\hline
\end{tabular}

basis of charge density or hydrophobicity. However, the functionality of particle systems depends not only on one or two but on several properties. The multidimensional character of the functional properties also requires a multidimensional separation approach. Usually, a series connection of different separation apparatuses is used for the multidimensional separation. This leads to an extended production time and a reduced product yield. The separation processes mentioned as examples also demonstrate the second challenge of separation technology. On the one hand, there are processes that exploit size and inertia effects. To achieve this, the particles must have a certain minimum size. As a rule of thumb, this is a particle size above $10 \mu \mathrm{m}$. On the other hand, for the separation processes based on diffusion effects and molecular interactions, the particles must not exceed a critical size of $\approx 100 \mathrm{~nm}$. Therefore, a separation gap arises in the range of particle sizes between $0.1 \mu \mathrm{m}$ and $10 \mu \mathrm{m}$ in which separation is very challenging. The process commonly referred to as magnetic seeded filtration [1] tackles these challenges by being multidimensional by nature and not limited by particle size. It aims to separate fine paramagnetic particles by agglomerating them with particles of higher magnetic susceptibility followed by a HGMS (high-gradient magnetic separation) step. Previous research found that not only ferromagnetic but also paramagnetic impurities could be separated by means of magnetic separation, which was explained by a hetero-agglomeration process between these particles [2]. The presented work is motivated by said findings and aims to explicitly investigate and enhance these effects. While many authors emphasize the HGMS part [3,2], this work takes a closer look at the first step: The hetero-agglomeration of ferro- and paramagnetic particles. In this context, both a computational and an experimental path should be taken.

As one can expect, an agglomeration process between different particles of different materials and sizes can be quite complex and hard to grasp mathematically. There is no fundamental equation solving this problem and hence model calculations have to be used in order to estimate process results. The most common way of doing so is to describe the process by a population balance equation. RAMKRISHNA broadly covers the basic theory of this topic in [4] and gives an overview about current and future applications and problems in [5]. A review of previous research specifically related to the modeling of agglomeration processes of colloidal systems is given in [6]. In this field, mainly discrete population balances are employed which generally start by initializing the distinct agglomerate classes. Starting with primary particles, all possible agglomerate combinations between them are created and are given characteristic class properties. Since the agglomeration process is non-stationary, a time-dependent change in each of these particle classes has to be calculated. As a result, population balance equations yield time- as well as agglomerate class-dependent concentration information. Many research groups have used this type of calculation in order to predict process results $[7,8]$, however none has given thorough information about the perhaps most crucial aspect of such calculations in hetero-agglomeration processes: the actual properties of a given agglomerate. When regarding a suspension of particles of different materials, said particles can vary severely in relevant properties, like for example surface charge or magnetic susceptibility. The main obstacle in such heterogeneous systems is that for each agglomerate, only one value can be used for each property. This work presents a method to deduce suitable values dependent on the composition of a given agglomerate by area-weighted collision case probabilities.

The second aspect of this work focuses on obtaining experimental results in order to compare, validate and discuss the theoretically gained information. There are plenty of works dealing with pure experimental investigations of hetero-agglomeration processes $[2,9,10]$. However, the time-dependent size distribution and agglomerate compositions are difficult and for the latter maybe even impossible to measure, which makes these works limited in their capability to fully describe the system. On the other hand, some research groups conducted purely theoretical studies of this problem [7] which yield the possibility of calculating plenty of non-measurable results. However, without validating these results through comparison with experimental data, these findings need to be taken with some skepticism. For this reason, many authors (including the authors of this work) recorded both experimental and theoretically gained data and are thus able to describe the hetero-agglomeration process from various angles $[1,8]$.

Due to the use of hydrophobic PVC particles, the influence of the hydrophobic interaction on the agglomeration process needs to be 
examined. However, to the authors' best knowledge, there is currently no work that implements these interactions into the population balance equation. This is mainly due to two reasons: first, the theoretical foundations of these interactions as yet have not been thoroughly understood and only empirical equations are available for describing them [11-14]. Second, these empirical equations require hard-to-measure material properties, which can generally only be obtained by AFM measurements. This work implements the hydrophobic interactions in the population balance and avoids AFM measurements by varying these hard-to-measure parameters until the calculated values match the experimental results. Further, both experimentally and theoretically gained findings are compared and validated for their physical meaningfulness. To sum up, this work strives for a new direction in experimental and theoretical hetero-agglomeration research. Experimental data is used to obtain information on hard-to-measure hydrophobic parameters and to validate the newly presented collision case model at a deeper physical level.

\section{Materials and methods}

\subsection{Experimental studies}

The particle systems used for the experiments are presented in Table 1 along with their characteristic properties. Note that as a measure for the mean diameter of these systems, the number-weighted median value $x_{50,0}$ is used since population balance equations operate on absolute particle numbers rather than volume fractions. The $\mathrm{pH}$ value in all experiments was 5.5, hence the zeta potential for all particle systems is given for this value. SEM images of the particle systems are given in Fig. 1. It can be seen that both particle systems have a round shape and similar particles size. For the continuous phase, ultrapure water with a residual ionic strength of $\approx 10^{-6} \frac{\mathrm{mol}}{\mathrm{L}}$ was used. The ionic strength was adjusted in the experiments by adding analytic grade sodium chloride.

The entire experiment can be split into four process steps which are illustrated by Fig. 2 and are described in the following section. Before conducting the actual agglomeration, the suspension had to be prepared by means of ultrasonic dispersion. First, the given amount of PVC particles and sodium chloride was weighted and added to $0.5 \mathrm{~L}$ of ultrapure water. Then, this suspension was dispersed with the Digital Sonifier 450 (Branson) for $20 \mathrm{~min}$ in order to break up existing PVC agglomerates. After the first dispersion step, a sample $\left(S_{0}\right)$ of this suspension was taken and stabilized with $0.5 \frac{\mathrm{g}}{\mathrm{L}}$ tetrasodium pyrophosphate in order to hinder agglomeration before measurement of this sample. Next, the iron particles were added and the suspension was further dispersed for $10 \mathrm{~min}$. The prepared suspension was then added to the vessel and stirred at the given speed $n$ for the given time $t_{A}$ in order to induce an agglomeration of the particles. After the agglomeration process, an iron matrix was lowered into the suspension and the whole tank was brought into a magnetic field. The magnetic field was produced by an openable Halbach permanent magnet [15] and had a magnetic field density of $0.35 \mathrm{~T}$. The matrix consists of a mesh of fine iron wires that produce large magnetic field gradients which then induce a strong magnetic force on the magnetic particles and hetero-agglomerates and thus ensure that these particles are collected by the matrix. The specific physical dependencies in HGMS are well described in the literature [16] and are therefore not extensively discussed in this work. Subsequently to the HGMS, the suspension containing the not separated particles and agglomerates was collected, dispersed with the sonifier for $10 \mathrm{~min}$ and stabilized with tetrasodium pyrophosphate. A sample of the residual suspension was then taken $\left(S_{i}\right)$ and analyzed together with the $S_{0}$ sample by means of absorbance spectroscopy at $\lambda=600 \mathrm{~nm}$. The validity of the Beer-Lambert law was ensured in previous studies, so that the absorbance value can be transferred into a concentration value. Under the assumption that all magnetic particles as well as all hetero-agglomerates (agglomerates containing magnetic particles) are separated in the HGMS step, a separation efficiency for the non-magnetic particles can be defined according to Eq. (1).

$T_{\mathrm{NM}}(t)=\left(1-\frac{A_{600}(t)}{A_{600}(0)}\right) \cdot 100 \%=\left(1-\frac{c_{\mathrm{NM}}(t)}{c_{\mathrm{NM}}(0)}\right) \cdot 100 \%$

In order to gain information about the influence of various processrelated parameters $(l)$, a design of experiment (DOE) approach was used. Five factors were varied on two levels in this study. The factors as well as the respective level values are presented in Table 2 . A full factorial DOE of the given problem yields $k=2^{l}=32$ parameter combinations that have to be conducted by experiment. However, by using a fractional factorial DOE, the amount of experiments can be reduced to $k=2^{l-1}=16$. The $2^{5-1}$ design still allows a clean resolution of all main and two-way interaction terms, since they are only aliased with interactions of an order larger than two [17]. The separation efficiencies for all 16 process parameter combinations were determined in triplicate and the mean separation efficiencies as well as the standard deviations were calculated. These values were then used in a multiple regression calculation in order to determine the effects of all factors and two-way interactions. A linear model as presented by Eq. (2) was used. The $X$ variables represent the factors given in Table 2 .

$T_{\mathrm{NM}}=\sum_{i=1}^{l} c_{i} X_{i}+\sum_{i=1}^{l-1} \sum_{j=i+1}^{l} c_{i, j} X_{i} X_{j}$

\subsection{Model calculations}

In this work, a population balance equation was used to model the hetero-agglomeration processes. At first, a discrete calculation grid has to be created containing all possible agglomerates in the given two material suspensions. A visualization of this process and the corresponding indexing are shown in Fig. 3.

The first index (i) represents the total number of primary particles in an agglomerate, while the second index $(j)$ represents the number of magnetic particles per agglomerate $(j-1)$. A 3-dimensional matrix $N(i$, $j, t)$ is then created in which the time-dependent number concentrations of all agglomerates are stored. This matrix is initialized for $t=0$ with the number concentration of primary particles $A_{1,1}$ and $A_{1,2}$ which can be calculated from given process parameters under the assumption of perfect spheres according to Eq. (3)

$N(1,2,0)=\frac{3 c_{v, M} V_{S}}{4 \pi r_{1,2}^{3}} \quad N(1,1,0)=\frac{3 \Phi_{v, N M} c_{v, M} V_{S}}{4 \pi r_{1,1}^{3}}$

where $r_{i, j}$ is the radius of an agglomerate and $V_{S}$ is the volume of the suspension. The actual time-discrete population balance is given in Eq. (4a).

Table 1

Particle systems used in experimental studies.

\begin{tabular}{lll}
\hline & Magnetic (M1) & Non-magnetic (NM1) \\
\hline Material & Iron (Fe) & $\begin{array}{l}\text { Polyvinyl chloride } \\
(\text { PVC) }\end{array}$ \\
Manufacturer & Merck KGaA, Darmstadt, & $\begin{array}{l}\text { Vinnolit GmbH, } \\
\text { Ismaning, Germany }\end{array}$ \\
Mean diameter $x_{50,0}$ & $1.07 \mu \mathrm{m}$ & $0.82 \mu \mathrm{m}$ \\
Density $\rho$ & $7870 \mathrm{~kg} \mathrm{~m}$ & $1400 \mathrm{~kg} \mathrm{~m}^{3}$ \\
Zeta potential $\zeta(\mathrm{pH}=5.5)$ & $\approx 0 \mathrm{mV}$ & $-32 \mathrm{mV}^{3}$ \\
Susceptibility $\chi$ & 10.13 & $4.6 \cdot 10^{-5}$ \\
Saturation magnetization & $17.7 \cdot 10^{5} \frac{\mathrm{A}}{\mathrm{m}}$ & - \\
$M_{\text {sat }}$ & $\left(H=1.75 \cdot 10^{5} \frac{\mathrm{A}}{\mathrm{m}}\right)$ & \\
Contact angle air, water $\Theta$ & $0^{\circ \mathrm{a}}$ & $91.7^{\circ} \pm 4.1^{\text {oa }}$
\end{tabular}

${ }^{\text {a }}$ Contact angles were measured by sessile drop method. Samples were prepared in a hydraulic press with constant force $(15 \mathrm{kN})$. 

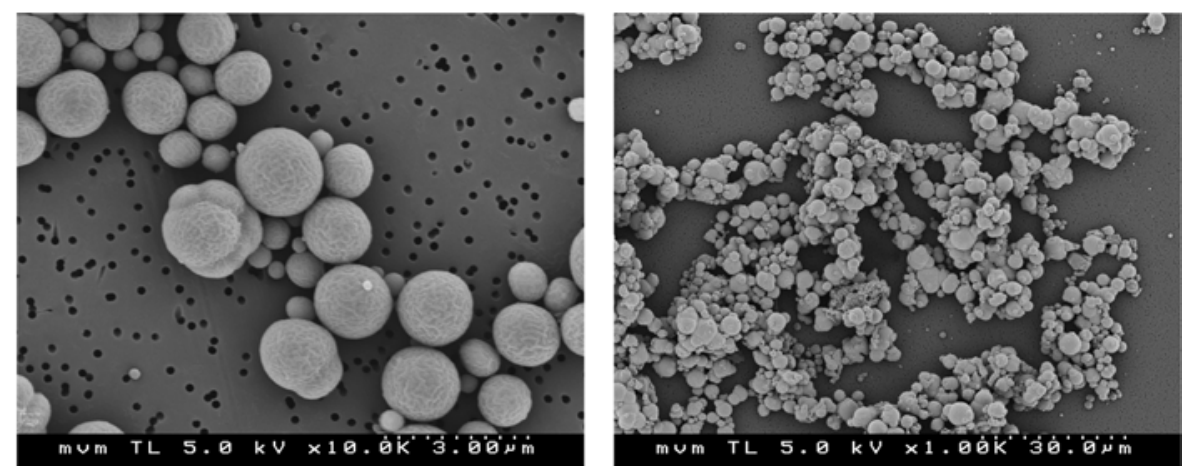

Fig. 1. SEM image of pure PVC particles (left) and agglomerates of PVC and iron particles (right).

$$
\begin{aligned}
N(i, j, t+1)= & N(i, j, t)+\Delta t(\underbrace{\frac{1}{2} \sum_{l=1}^{i-1} \sum_{m=1}^{j} N(l, m, t) N(i-l, j-m, t) F_{\operatorname{lm},(i-l)(j-m)}}_{(1)} \\
& -\underbrace{\sum_{l=1}^{N_{S} \max ^{-i}} \sum_{m=1}^{l+1} N(i, j, t) N(l, m, t) F_{\mathrm{ij}, \mathrm{lm}}}_{(2)})
\end{aligned}
$$

$F_{\mathrm{ij}, \operatorname{lm}}=\alpha_{\mathrm{ij}, \operatorname{lm}} \beta_{\mathrm{ij}, \operatorname{lm}}$

The agglomeration efficiency $F_{i j, l m}$ is given by Eq. (4b) and describes the agglomeration rate between agglomerates $A_{i, j}$ and $A_{l, m}$. It is the product of the agglomeration efficiency $\alpha$ and the collision frequency $\beta$. Vividly described, this means that particles that agglomerate first have to come into contact (probability given by $\beta$ ) and then have to overcome an energy barrier and "stick" (probability given by $\alpha$ ).

In order to find further expressions for $\alpha$ and $\beta$, a process distinction has to be made: The equations depend on whether the process is diffusion- or flow-controlled (peri- or orthokinetic). The PÉCLET number presented in Eq. (5) describes the orthokinetic transport in relation to the perikinetic one and can thus be used to determine the given conditions $[8]$

$\mathrm{Pe}=\frac{2 \sqrt{3}\left(r_{i, j}+r_{l, m}\right)^{2} r_{i, j} G \pi \mu}{\mathrm{kT}\left(1+R^{-1}\right)}$

where $G$ is the mean shear rate in the system, $\mu$ the viscosity of the fluid and $R$ is the size ratio of both particles. In the scope of this work, the PÉCLET number roughly varied between $10^{1}$ and $10^{2}$, however, an accurate calculation is difficult due to the undefined energy dissipation by the impeller. This proposes an interesting situation: Generally, $P e>1$
Table 2

Varied factors with respective levels.

\begin{tabular}{lllll}
\hline \multirow{2}{*}{ Name } & Description & Unit & \multicolumn{2}{l}{ Factor level } \\
\cline { 4 - 5 } & & & - & + \\
\hline$n$ & Rotary speed & {$\left[\mathrm{min}^{-1}\right]$} & 100 & 250 \\
$c_{v, M}$ & Volume concentration magnetic particles & {$[\mathrm{vol} \%]$} & 0.005 & 0.01 \\
$\Phi_{v, \mathrm{NM}}$ & Volume concentration ratio non-magnetic & {$[-]$} & 0.5 & 2 \\
& particles $\Phi_{v, \mathrm{NM}}=\frac{c_{v, \mathrm{NM}}}{c_{v, M}}$ & & & \\
$t_{A}$ & Agglomeration time & {$[\mathrm{min}]$} & 10 & 30 \\
$I$ & Ionic strength & {$\left[\frac{\mathrm{mol}}{\mathrm{L}}\right]$} & $10^{-6}$ & $10^{-3}$ \\
& & & & \\
\hline
\end{tabular}

indicates an orthokinetic process, however, in this range of values, diffusion processes may not be neglected entirely. Since the collision between particles is likely to be flow-controlled, the collision frequency $\beta$ can be calculated according to Eq. (6) [8].

$\beta_{\mathrm{ij}, \mathrm{lm}}=2.3\left(r_{i, j}+r_{l, m}\right)^{3} G$

The calculation of the collision efficiency however, is more challenging. In the case of orthokinetic agglomeration, a trajectory analysis needs to be conducted in order to include the influence of the inertia of the particles. Various authors implemented this type of calculation in their population balances $[8,1]$. However, since the PÉCLET number does not indicate pure orthokinetic conditions, the FucHs approach for perikinetic agglomeration [18] is used in this work. Thereby, the collision efficiency is attained by integrating the interaction potential between two particles. The implemented expression is given in Eq. (7)
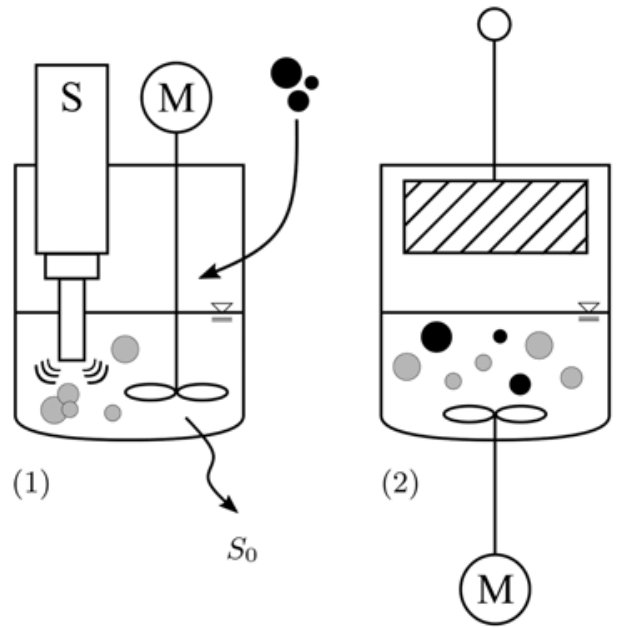
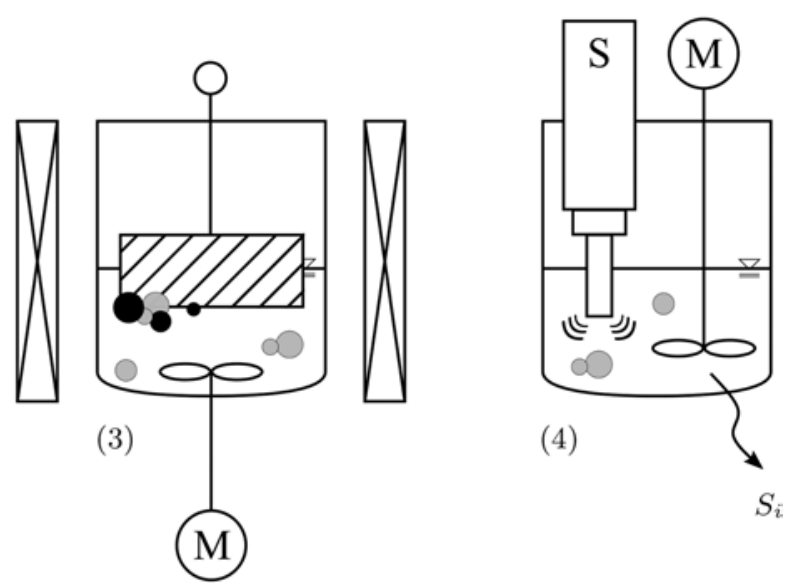

Fig. 2. Illustration of the four process steps. (1): Suspension preparation. (2): Agglomeration (no magnetic field, iron matrix outside suspension). (3): Separation (magnetic field, iron matrix in suspension). (4): Post processing (breakup of PVC agglomerates). 


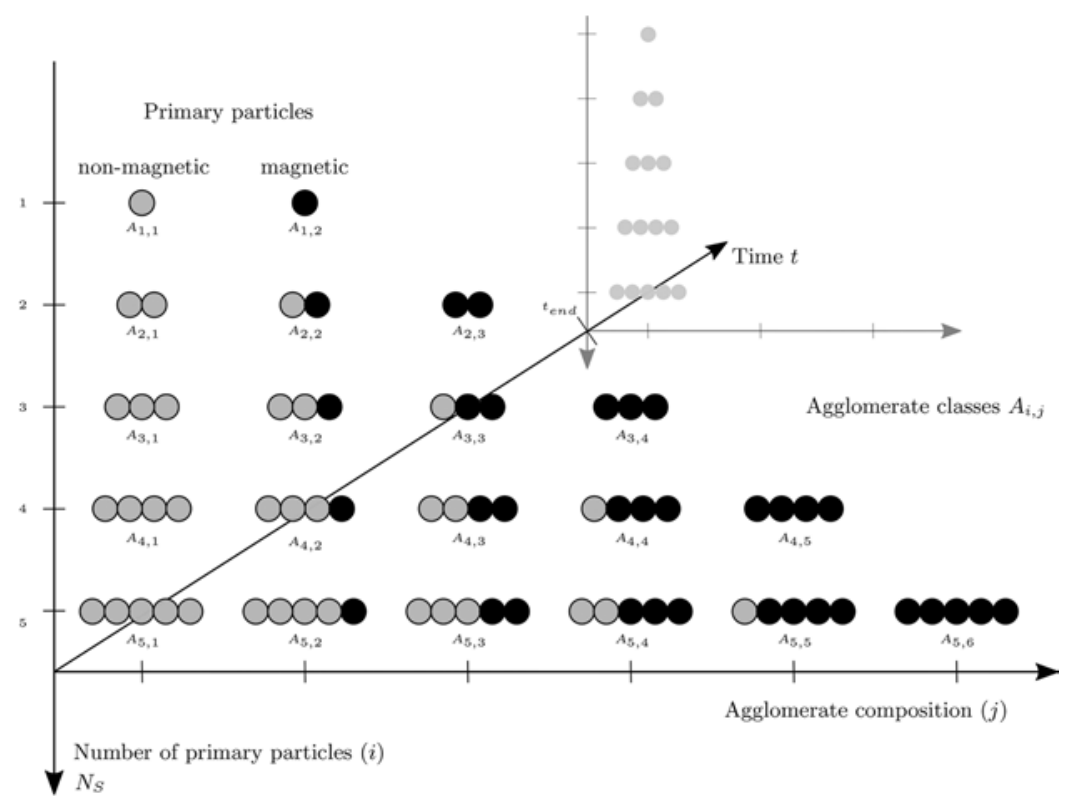

Fig. 3. Schematic figure of all possible agglomerate combinations for $N_{S, \max }=5$. The time dependency of the number concentration matrix $N$ is represented in the third dimension ( $t$-axis).

$\alpha_{\mathrm{ij}, \mathrm{lm}}=\left[\left(1+\frac{r_{i, j}}{r_{l, m}}\right) \int_{1+\frac{r_{i, j}}{r_{l, m}}}^{\infty} \frac{D_{\infty}}{D_{\mathrm{ij}, \mathrm{lm}}} \exp \left(\frac{\Psi_{\Sigma}}{\mathrm{kT}}\right) \frac{\mathrm{ds}}{s}\right]^{-1}$

where $s$ is the dimensionless center-to-center distance [7]. Here, the HONIG approach [19] in Eq. (8) is used to account for viscous effects

$\frac{D_{\infty}}{D_{\mathrm{ij}, \operatorname{lm}}}=\frac{6(s-2)^{2}+13(s-2)+2}{6(s-2)^{2}+4(s-2)}$

The expression $\Psi_{\Sigma}$ in Eq. (7) represents the sum of all interaction energies between the two agglomerating particles. The classical approach of the DLVO theory only regards van-der-Waals $\left(\Psi_{v d W}\right)$ and electrostatic $\left(\Psi_{e l}\right)$ interactions [20]. This work however, also considers hydrophobic $\left(\Psi_{h p h}\right)$ interactions and a mathematical expression for each interaction energy is given in the following paragraph.

The van-der-Waals interaction energy is given by Eq. (9) which is valid for the interaction of two spheres [21]

$$
\begin{aligned}
\Psi_{\mathrm{vdW}, \mathrm{ij}, \operatorname{lm}}(h)= & -\frac{A_{H}}{6}\left[\frac{2 r_{i, j} r_{l, m}}{h^{2}+2 r_{i, j} h+2 r_{l, m} h}+\frac{2 r_{i, j} r_{l, m}}{h^{2}+2 r_{i, j} h+2 r_{l, m} h+4 r_{i, j} r_{l, m}}\right. \\
& \left.+\ln \left(\frac{h^{2}+2 r_{i, j} h+2 r_{l, m} h}{h^{2}+2 r_{i, j} h+2 r_{l, m} h+4 r_{i, j} r_{l, m}}\right)\right]
\end{aligned}
$$

where $A_{H}$ is the HAMAKER constant and $h$ represents the absolute surfaceto-surface distance which can be calculated from the dimensionless center-to-center distance $s$ according to Eq. (10).

$h(s)=\frac{s\left(r_{i, j}+\eta_{, m}\right)}{2}-r_{i, j}-\eta_{, m}$

The electrostatic interaction energy can be calculated by Eq. (11a) [21]

$\Psi_{\mathrm{el}, \mathrm{ij}, \mathrm{lm}}=\frac{128 \pi r_{i, j} \eta_{l, m} I N_{a} \mathrm{kT}}{\left(r_{i, j}+\eta_{, m}\right) \kappa^{2}} \tanh \left(\frac{e \varphi_{i, j}}{4 \mathrm{kT}}\right) \tanh \left(\frac{e \varphi_{l, m}}{4 \mathrm{kT}}\right) \exp (-\kappa h)$

$\kappa=\sqrt{\frac{2 e^{2} \mathrm{IN}_{a}}{\epsilon \mathrm{KT}}}$

where $N_{a}$ is the AVOGADRo number, $e$ is the electron charge, $\varphi$ is the surface potential of the particles and $\kappa$ is the reciprocal DEBYE length which can be calculated by Eq. (11b). As the surface potential is not measurable directly, the zeta potential $\xi$ is used as an approximation.

Since the PVC particles exhibit a contact angle of $\Theta>90^{\circ}$, they can be regarded as fully hydrophobic and thus hydrophobic interactions need to be considered [14]. For these interactions, an empirical bi-exponential model derived in [12] is used and given in Eq. (12)

$\Psi_{\mathrm{hph}, \mathrm{ij}, \mathrm{lm}}=-\frac{r_{i, j}+r_{l, m}}{2}\left[C_{1} d_{1} \exp \left(\frac{-h}{d_{1}}\right)+C_{2} d_{2} \exp \left(\frac{-h}{d_{2}}\right)\right]$

where $C_{1}$ and $C_{2}$ are the parameters adjusting the strength and $d_{1}$ and $d_{2}$ are the parameters adjusting the range of the short-ranged and longranged hydrophobic interactions. [12] has shown that this model is able to describe various reported AFM datasets of the still not entirely understood hydrophobic interaction between particles. Note that this attractive interaction has not only been shown to be present between two hydrophobic particles, but also between hydrophobic and hydrophilic particles and therefore, this interaction may also arise between PVC and iron particles.

Lastly, all interaction energies are summed up according to Eq. (13) and used in Eq. (7) to compute the value of the collision efficiency $\alpha$.

$\Psi_{\Sigma}=\Psi_{\mathrm{vdW}}+\Psi_{\mathrm{el}}+\Psi_{\mathrm{hph}}$

However, in order to conduct any calculation, the material properties of all agglomerate classes have to be known, which can be a large obstacle to overcome. In this work, a different and new approach was used solely based on the material data of the primary particles $A_{1,1}$ and $A_{1,2}$ and the surface-related agglomerate composition. For a given agglomerate $A_{i, j}$, the surface-related proportion of non-magnetic particles is calculated by Eq. (14) and stored in matrix $Y_{N M}$. This model assumes perfect spheres as well as an agglomerate porosity of 0 . Note that even without these assumption, an agglomerate may be larger in size and surface area, however, the surface proportions of both primary particle classes are likely to be similar.

$Y_{\mathrm{NM}}(i, j)=\frac{(i-(j-1)) r_{1,1}^{2}}{(i-(j-1)) r_{1,1}^{2}+(j-1) r_{1,2}^{2}}$

The main question is to what extent each of the two agglomeration partners exhibits non-magnetic or magnetic behavior. Depending on this, four cases can be derived and are presented in Fig. 4. The cases differ in the fact whether agglomerates $A_{i, j}$ and $A_{l, m}$ collide with their non-magnetic or magnetic surface. The calculation assumes that the probability of an agglomerate $A_{i, j}$ colliding with the non-magnetic part of its surface is given only by the surface fraction $Y_{N M}(i, j)$ and no 
further flow- or material-related property of any kind. Since the matrix $Y_{N M}$ is known, the probability for each collision case between two agglomerates $A_{i, j}$ and $A_{l, m}$ can be calculated according to the equations given in Fig. 4. Furthermore, for each of the four cases, the surface properties of both agglomeration partners are known and can be expressed by the pure material properties of either the non-magnetic or magnetic primary particles (depending on the case). Thus, for each case, a corresponding collision efficiency $\alpha_{A-D}$ can be calculated. Since all case probabilities and collision efficiencies are known, a surface area-weighted collision efficiency can be calculated according to Eq. (15).

$\alpha_{\mathrm{ij}, \mathrm{lm}}=P_{A} \alpha_{A}+P_{B} \alpha_{B}+P_{C} \alpha_{C}+P_{D} \alpha_{D}$

This method is an alternative to volume-weighted averaging of material properties for each agglomerate and then using these properties to directly calculate one collision efficiency. However, from a physical perspective, averaging of e.g. surface potentials does not seem fit for a surface-controlled process. For better illustration of this fact, the following example is presented. Consider an agglomerate consisting of one PVC and one Fe particle of equal size and shape. When averaging the material properties by volume, this agglomerate has a zeta-potential of $\zeta=-16 \mathrm{mV}$ regardless of the collision cases presented in Fig. 4. A collision arising between two of these agglomerates always shows a non-negligible electrostatic repulsion, while in reality, three of the four equally probable collision cases (A, B and D) should not exhibit any due to the non-charged iron surface. This has a tremendous effect on the dynamics of the whole population balance which is further intensified the more heterogeneous the material properties of the primary particles are. Therefore, the selected particle systems with their large differences in material properties are well suited to critically examine the presented collision case model.

For the calculations, three different parameter groups can be distinguished which are given in Table 3. Regarding the constant parameters, especially the maximum number of primary particles per agglomerate $N_{S, \max }$ and the time step size $\Delta t$ are of interest and demand further investigation. $N_{S, \max }$ defines the agglomeration grid and thus the number of calculations which need to be performed per time step. However, a too small value of $N_{S, \max }$ may influence the calculation result, since it also limits the possible agglomerate combinations and therefore limits further agglomeration. The time step size $\Delta t$ defines the number of discrete calculations that have to be conducted until the predefined agglomeration time $t_{A}$ is reached. As in every discrete method, a too small step size increases the real-world calculation time, while too large steps may lead to non-physical results and divergence. In preliminary studies, both grid-relevant parameters have been investigated. The results are shown in Fig. 5. From Fig. 5a, it can be seen that both the relative change in separation efficiency $d T / T$ as well as the calculation time $t$ are dependent on $N_{S, \max }$ in an exponential manner. A trade-off between calculation time and accuracy was found with $N_{S}=10$. Fig. $5 \mathrm{~b}$ shows that the relative change of calculation results starts to rise at approximately $\Delta t=1 \mathrm{~s}$ and diverges strongly for large time steps. Therefore, this parameter setting was chosen for the calculations.

The second set of parameters are the process parameters given by the DOE in Table 2. They ensure comparability between model calculations and experimental results. As mentioned above, 16 process-related parameter combinations are investigated experimentally and therefore all 16 of these combinations are calculated by the population balance.

The third parameter group contains model parameters which are varied between calculations. This parameter group mainly contains hard-to-measure material properties such as the parameters of the hydrophobic interaction in Eq. (12) or hard-to-measure process parameters expressed in correction terms such as a correction term for the collision frequencies $\beta$. This opens up an optimization problem: The model parameters can be varied in a way such that the calculated separation efficiencies best match the measured ones. Therefore, a second, full factorial DOE was conducted in order to find the optimal model parameters. Table 4 gives an overview of the varied factors and the range in which their levels were varied. Note that not only two levels $(+$ and -$)$ were investigated but multiple values in between. For the strength parameters of the hydrophobic interaction, a further differentiation was conducted: All four different collision cases are assigned different hydrophobic parameters as it can be expected that two PVC surfaces (NM-NM) interact differently than an iron and a PVC surface (M-NM). However, for the interaction between two iron surfaces, no hydrophobic interaction is expected and thus both parameters $C_{1, M, M}$ and $C_{2, M, M}$ are set to zero. With each model parameter combination, the full experimental DOE (16 experiments) as well as the resulting root-mean-square error (RMSE) given in Eq. (16) were calculated.

$\operatorname{RMSE}=\sqrt{\frac{1}{16} \sum_{i=1}^{16}\left(T_{\mathrm{NM}, \exp , i}-T_{\mathrm{NM}, \bmod , i}\right)^{2}}$

By comparing the RMSE value of the various model parameter combinations, an optimum parameter set can be found to best describe the given system. Thus, the combination of experiment and model calculation allows to gain extra hard-to-measure information about the material properties of the particle systems.

\section{Results and discussion}

\subsection{Experimental results}

Preliminary studies showed that a separation efficiency can be measured even when no magnetic particles are added to the system. This effect is due to systematic errors such as sedimentation effects and cannot be credited towards a hetero-agglomeration process. In order to identify, quantify and ultimately correct this effect, the agglomeration kinetics of the process only containing non-magnetic particles as well as the total process containing non-magnetic and magnetic particles were measured. The results are shown in Fig. 6. In order to quantify the

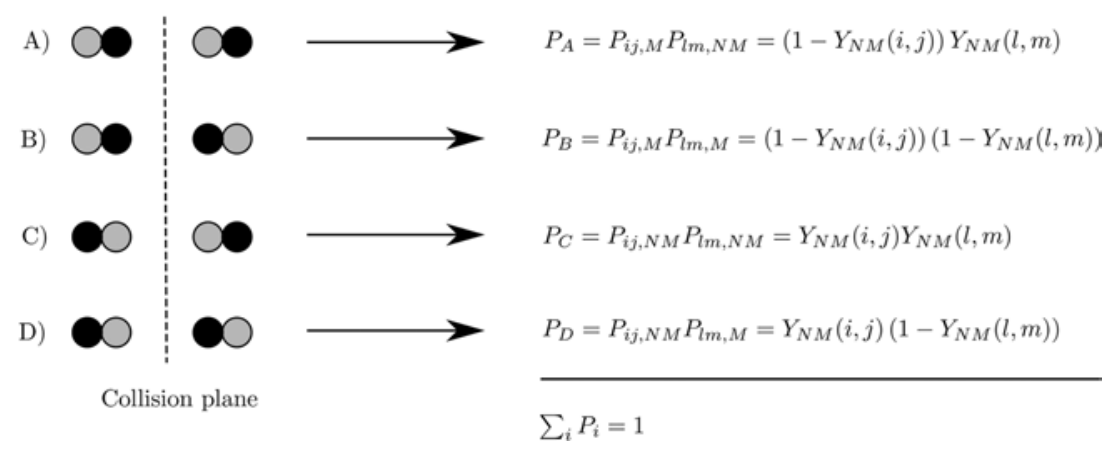

Fig. 4. Various collision cases and corresponding probability calculations for the agglomeration of $A_{i, j}$ with $A_{l, m}$. 
Table 3

Parameter groups and examples.

\begin{tabular}{lll}
\hline Parameter group & Examples & Meaning for calculation \\
\hline Constant parameters & $N_{S, \max }, \Delta t, r_{1,1}, r_{1,2}, \varphi_{1,1}, \varphi_{1,2}, A_{H, 1,1}, A_{H, 1,2}, \ldots$ & $\begin{array}{l}\text { Not changed during calculations. Mainly measurable/known material properties of the primary } \\
\text { particles. }\end{array}$ \\
$\begin{array}{ll}\text { Process parameters } \\
\text { Model parameters }\end{array}$ & $\begin{array}{l}n, c_{v, M}, \Phi_{v, N M}, t_{A}, I \\
C_{1, N M, N M}, C_{2, N M, N M}, C_{1, M, N M}, C_{2, M, N M}, d_{1}, d_{2}, \beta_{c o r r, 100},\end{array}$ & $\begin{array}{l}\text { Parameters given by the DOE of the experimental studies. } \\
\text { Adjustable model parameters. Mainly hard-to-measure material properties of the primary } \\
\text { particles. }\end{array}$ \\
\hline
\end{tabular}

effects, a mathematical analysis of the problem was conducted under simplifying assumptions starting from a reduced agglomeration kinetic given in Eq. (17).

$\frac{\mathrm{dN}_{i, j}}{\mathrm{dt}}=N_{i}(t) N_{j}(t) \alpha_{i, j} \beta_{i, j}=N_{i}(t) N_{j}(t) c_{i, j}$

Assuming the separation in the case of pure PVC to be due to a homo-agglomeration process, this equation can be rewritten and further used to calculate a separation efficiency according to Eq. (18a). By fitting this equation to the measured kinetic, the coefficient $c_{\mathrm{Hom}}$ is received. When describing a pure hetero-agglomeration process, the equation cannot be solved readily because the number of magnetic particles $N_{M}$ is unknown. Since a hetero-agglomerate can still agglomerate further (and thus act as a magnetic particle), it can be assumed $N_{M}=$ const which yields an integratable equation and the expression for the pure hetero-agglomeration process given in Eq. (18b). In order to obtain the coefficient $c_{\mathrm{Het}}$, the total system in which homo and heteroagglomeration compete has to be investigated. Note that the measured kinetic in Fig. 6 describes exactly that. By adding both rates and integrating the resulting expression, Eq. (18c) is obtained. Since $c_{\mathrm{Hom}}$ is already known, $c_{\mathrm{Het}}$ can be calculated by fitting Eq. (18c) to the measured kinetic. This coefficient can then be used to compute the corrected value for $T_{N M \text {,Het }}$ according to Eq. (18b). When conducting an experiment, only the separation efficiency at $t=t_{A}$ is determined rather than the whole kinetic. This datapoint is represented in Fig. 6 by the bold points at $t=30 \mathrm{~min}$. Nevertheless, the investigation of the agglomeration kinetics showed that the derived models represent the measured curves well and so the above-described procedure can still be used to obtain the coefficients $c_{\mathrm{Hom}}$ and $c_{\mathrm{Het}}$ for any given experimental result. The latter coefficient is then inserted into Eq. (18c) to calculate the corrected value of $T_{N M, H e t}\left(t=t_{A}\right)$. The solid line in Fig. 6 thus represents the corrected pure hetero-agglomeration process. However, for this calculation, the experimental results for the pure homo-agglomeration process are required. They were determined experimentally in a separate DOE.

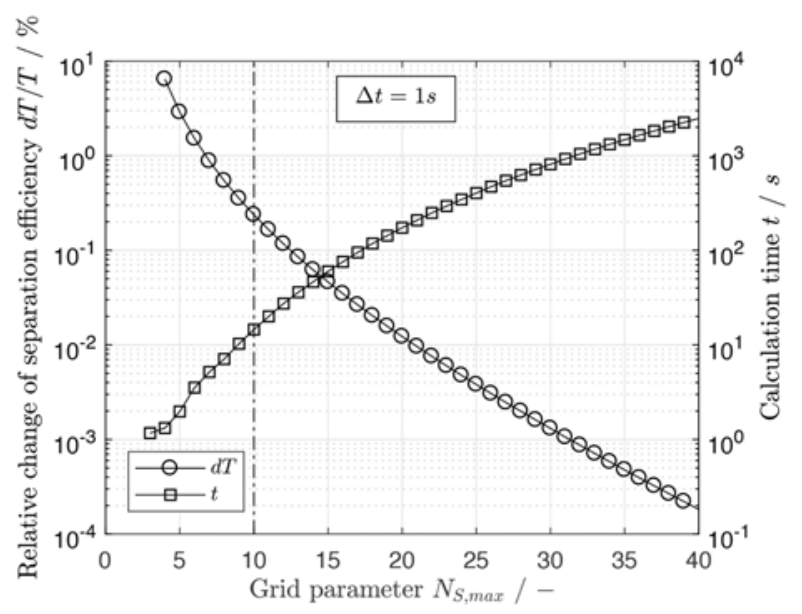

(a) Variation of grid size $N s(\Delta t=c o n s t)$.

$$
\begin{aligned}
\frac{\mathrm{dN}_{\mathrm{NM}}}{\mathrm{dt}} & =-c_{\mathrm{Hom}}^{*} N_{\mathrm{NM}}^{2} \\
& \longrightarrow T_{\mathrm{NM}, \mathrm{Hom}}(t)=\left(1-\frac{1}{1+c_{\mathrm{Hom}} t}\right) \cdot 100 \%
\end{aligned}
$$

$$
\begin{aligned}
\frac{\mathrm{dN}_{\mathrm{NM}}}{\mathrm{dt}} & =-c_{\mathrm{Het}}^{*} N_{\mathrm{NM}} N_{M}=-c_{\mathrm{Het}} N_{\mathrm{NM}} \\
& \longrightarrow T_{\mathrm{NM}, \mathrm{Het}}(t)=\left(1-\exp \left(-c_{\mathrm{Het}} t\right)\right) \cdot 100 \%
\end{aligned}
$$

$$
\begin{aligned}
\frac{\mathrm{d} N_{\mathrm{NM}}}{\mathrm{dt}} & =-c_{\mathrm{Het}} N_{\mathrm{NM}}-c_{\mathrm{Hom}}^{*} N_{\mathrm{NM}}^{2} \\
& \longrightarrow T_{\mathrm{NM}, \mathrm{Tot}}(t)=\left(1-\frac{c_{\mathrm{Het}}}{\left(c_{\mathrm{Hom}}+c_{\mathrm{Het}}\right) \exp \left(c_{\mathrm{Het}} t\right)-c_{\mathrm{Hom}}}\right) \cdot 100 \%
\end{aligned}
$$

After correcting the results in the above-described physically rational manner, they were used in a linear multiple regression model in order to extract the relevant parameters for this process. The absolute effects of the factors on the separation efficiency are shown in Fig. 7a. It can be seen that the rotational speed $n$ and thus the collision efficiency has the largest effect in the investigated range of parameters. This indicates a destabilized system in which agglomeration is only limited by collision. Furthermore, the interplay between the positive effect of $c_{v, M}$ and the negative effect of $\Phi_{v, \mathrm{NM}}$ indicates that for a good agglomeration result, as many magnetic particles as possible should be agglomerated with as few non-magnetic particles as possible. Again, this effect can be ascribed to the collision-controlled mechanism, since the probability of a non-magnetic particle colliding with a magnetic one is increased. The positive effect of the agglomeration time is self-explanatory, while the non-existent effect of ionic strength can be credited towards the noncharged surface of the magnetic particles and thus the subordinate role of the electrostatic interactions. Generally, the experimental separation efficiencies varied in a range from $T_{N M, \min }=5.93 \%$ for the worst to $T_{N M, \max }=86.13 \%$ for the best process parameter combination. This demonstrates that the selected parameter range is able to cover a large area of possible separation efficiencies and that the magnetic seeded filtration process itself is capable of achieving a high and desired result. The experimental results showed a rather minor mean standard

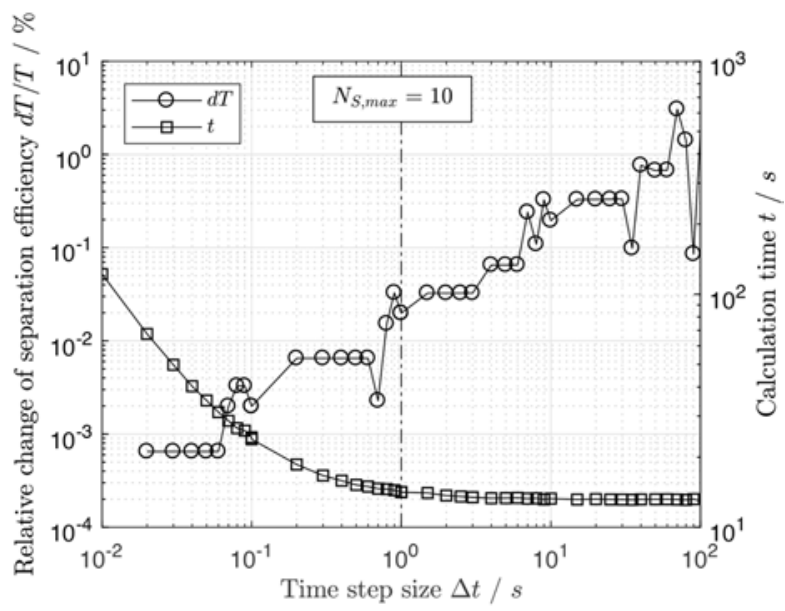

(b) Variation of discrete time step size $\Delta t(N s=$ const $)$.

Fig. 5. Study of grid relevant parameters $N s$ and $\Delta t$. 
Table 4

Varied model parameters, with investigated range and optimum value.

\begin{tabular}{|c|c|c|c|c|c|}
\hline \multirow[t]{2}{*}{ Name } & \multirow[t]{2}{*}{ Description } & \multirow[t]{2}{*}{ Unit } & \multicolumn{2}{|c|}{ Factor range } & \multirow[t]{2}{*}{ Optimum } \\
\hline & & & - & + & \\
\hline$C_{1, N M, N M}$ & Strength parameter short-ranged hydrophobic interaction between NM and NM & {$\left[\frac{\mathrm{mN}}{\mathrm{m}}\right]$} & 0 & 300 & * \\
\hline$C_{2, N M, N M}$ & Strength parameter long-ranged hydrophobic interaction between NM and NM & {$\left[\frac{\mathrm{mN}}{\mathrm{m}}\right]$} & 0 & 10 & * \\
\hline$C_{1, M, N M}$ & Strength parameter short-range hydrophobic interaction between $\mathrm{M}$ and NM & {$\left[\frac{\mathrm{mN}}{\mathrm{m}}\right]$} & 0 & 300 & $*$ \\
\hline$C_{2, M, N M}$ & Strength parameter long-ranged hydrophobic interaction between $\mathrm{M}$ and NM & {$\left[\frac{\mathrm{mN}}{\mathrm{m}}\right]$} & 0 & 10 & * \\
\hline$d_{1}$ & Decay length short-ranged hydrophobic interaction & {$[\mathrm{nm}]$} & 1 & 10 & $\leq 2$ \\
\hline$d_{2}$ & Decay length long-ranged hydrophobic interaction & {$[\mathrm{nm}]$} & 3 & 30 & $\leq 4$ \\
\hline$\beta_{\text {corr }, 100}$ & Correction term collision frequency $n=100 \mathrm{rpm}$ & {$[-]$} & 1 & 15 & 7.4 \\
\hline$\beta_{c o r r, 250}$ & Correction term collision frequency $n=250 \mathrm{rpm}$ & {$[-]$} & 1 & 15 & 9 \\
\hline
\end{tabular}

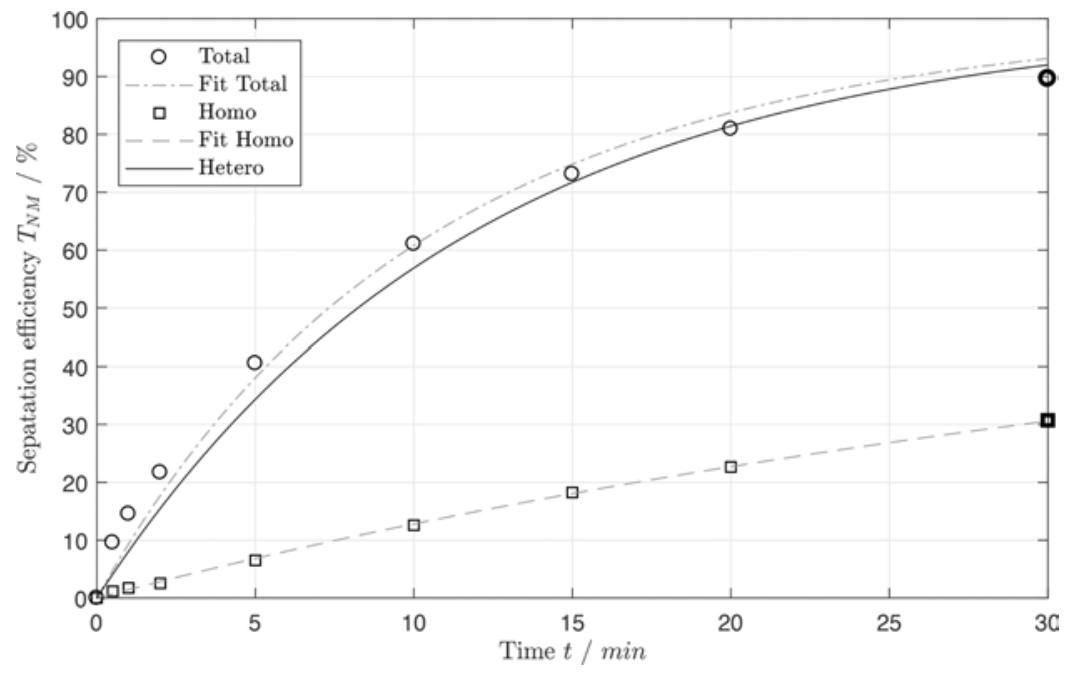

Fig. 6. Experimentally measured agglomeration kinetics of a pure PVC suspension (homo-agglomeration), the total process containing magnetic and non-magnetic particles as well as the resulting calculated pure hetero-agglomeration. Process parameters are $n=250 \mathrm{rpm}, c_{v, M}=0.01 \mathrm{vol} \%, \Phi_{v, \mathrm{NM}}=1$ and $I=10^{-3} \frac{\mathrm{mol}}{\mathrm{L}}$.

deviation of $\bar{\sigma}_{\text {exp }}=3.75 \%$ and good accordance with the agglomeration theory and are thus further used to conduct the comparison between model calculations and experimental data.

\subsection{Model adjustment and validation}

As described above, a DOE for the calculation in which the model parameters were varied was conducted. The parameter combination

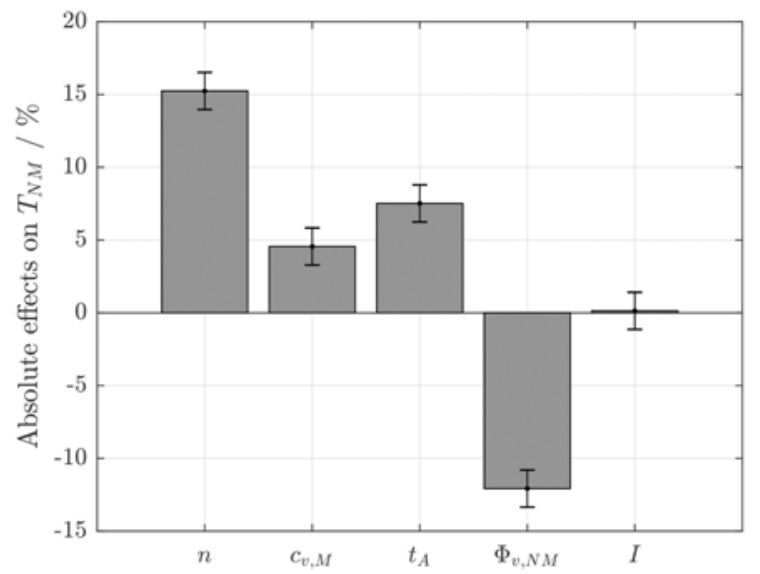

(a) Experimental study displaying the least RMSE values is given in Table 4. The discussion of this optimal parameter setup is performed in a separate section below. The resulting root-mean-square error is $R M S E=13.37 \%$, while the mean absolute error is $M A E=11.49 \%$. Note that the RMSE weights large residuals more severely and is therefore larger in absolute value. At first sight, the mean residuals appear to be disproportional to the mean standard deviation of the experiments and thus indicate a poor fit between experiments and model calculations. However, only 16

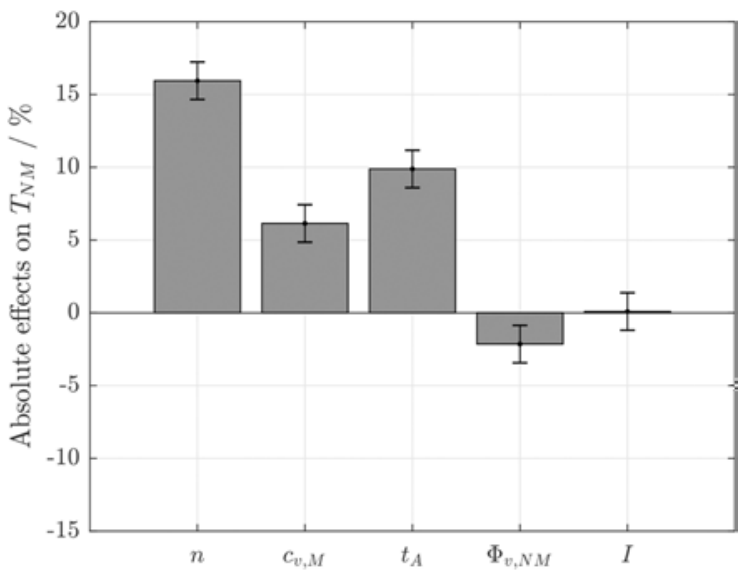

(b) Model calculations

Fig. 7. Absolute effects of the process parameters on the separation efficiency. 
singular parameter combinations were investigated and it appears to be more meaningful to compare physical effects rather than absolute errors. Therefore, the calculated results were used in a multiple regression model to identify the effects of the factors on the separation efficiency. The effects are shown in Fig. $7 \mathrm{~b}$ and indicate a good agreement with the experimental results: The effects of $n, c_{v, M}, t_{A}$ and $I$ are nearly identical in direction and absolute value, while only $\Phi_{v, \mathrm{NM}}$ does not show the same influence as in the experimental study. This difference in one parameters effect can explain the rather large RMSE value, since this effect produces large residuals for some of the 16 investigated parameter combinations. The reason for this deviation in effects cannot be resolved with absolute certainty, but a possible explanation lies in a systematic error in the experiments overestimating the influence of $\Phi_{v, \mathrm{NM}}$. Generally, it can be argued that by fitting model parameters to experimental data, it is not meaningful to further compare the calculated to the experimental results since they need to show good agreement by nature. The answer to this critical question is ambiguous: Yes, the absolute values of the target value need to show accordance. However, the physical causalities of gaining these results are not necessarily identical since an infinite number of model equations can lead to the same result without considering any agglomeration theory. In conclusion, it can therefore be stated that the comparison between Fig. $7 \mathrm{a}$ and $\mathrm{b}$ shows the capability of the model to portray the main physical correlations and said model can thus be seen as validated on a basic theoretical level although the absolute values may show nonnegligible residuals.

Another way of verifying the physical soundness of the calculations is by examining the agglomeration kinetics. The model offers the possibility of knowing the exact agglomerate distribution for any given point of the experiment and thus the separation kinetic can be extracted readily. As mentioned above, the absolute values of the separation efficiencies at $t=t_{A}$ can show rather large differences to the experimental values which makes a direct comparison difficult. From a physical standpoint, the shapes or the slopes of the curves are more interesting than the final value. Therefore, the measured and calculated kinetics were normalized to the final point at $t=t_{A}$ and the results are shown in Fig. 8. Both graphs exhibit the same exponential character although the model calculation overall shows a larger separation efficiency. This can be explained by the earlier discussed rivalry between homo- and heteroagglomeration in the experiments resulting in a particle loss not accountable for in the pure hetero-agglomeration kinetic. Nevertheless, Fig. 8 shows that the model calculations are able to reproduce real measured agglomeration kinetics.

\subsection{Discussion}

After verifying the general credibility of the model calculations, the results of the model parameter optimization deserve a closer investigation. Especially estimates of the hard-to-measure material properties of the hydrophobic interaction are now accessible through this calculation. The optimization results showed that the hydrophobic parameters of the interaction between iron and PVC surfaces $\left(C_{1, M, N M}\right.$ and $C_{2, M, N M}$ ) do not affect the dynamic or result of the calculations and are thus marked with * in Table 4 . This is due to the fact that this interaction exhibits no electrostatic repulsion. Therefore, this sub-process is only limited by the collision frequency and independent of further attractive potentials. Furthermore, the interaction between two PVC surfaces rather showed an optimum parameter range than a specific set of optimum parameter values. Hereby, the strength parameters $C_{1, N M, N M}$ and $C_{2, N M, N M}$ played a subordinate role compared to the decay lengths $d_{1}$ and $d_{2}$ in this context: the calculations only exhibited reasonable residuals with parameter values of $d_{1}$ and $d_{2}$ in the range given in Table 4, while for these decay lengths, the absolute values of $C_{1, N M, N M}$ and $C_{2, N M, N M}$ did not influence the outcome significantly. A closer look at the interaction potentials between two PVC surfaces revealed that these values mark the range in which the hydrophobic interaction between two PVC surfaces is surpassed by the electrostatic repulsion viz. in which PVC surfaces are stable and do not agglomerate. Fig. 9a shows the potential curves for the critical model parameter setup and maximal ionic strength resulting in a minimal range of the electrostatic repulsion. An energy barrier is apparent which prevents particles from agglomerating until the surface distance becomes extremely small. However, as shown in Fig. 9b, even a small increase of the decay lengths $d_{1}$ and $d_{2}$ dissolves the energy barrier and the system becomes destabilized. These results indicate that in the given system, all interactions between iron and another surface (iron or PVC) are fully destabilized, while interactions between two PVC surfaces do not lead to an agglomeration. This means that the most drastic differences in agglomeration-relevant material properties are present. As outlined above, this setup thus challenges the collision case model to the extreme and since it was nevertheless able to produce physically sound results, the overall credibility of the validation is underlined.

With regard to the uniqueness of the parameter set found, it can be stated that the displayed combination should be regarded as best fit and not as an exact solution. As already mentioned, for many parameters an optimal range rather than an optimal value was observed, which further indicates that the optimization procedure provides a general estimate rather than exact values. Nevertheless, the method presented shows the

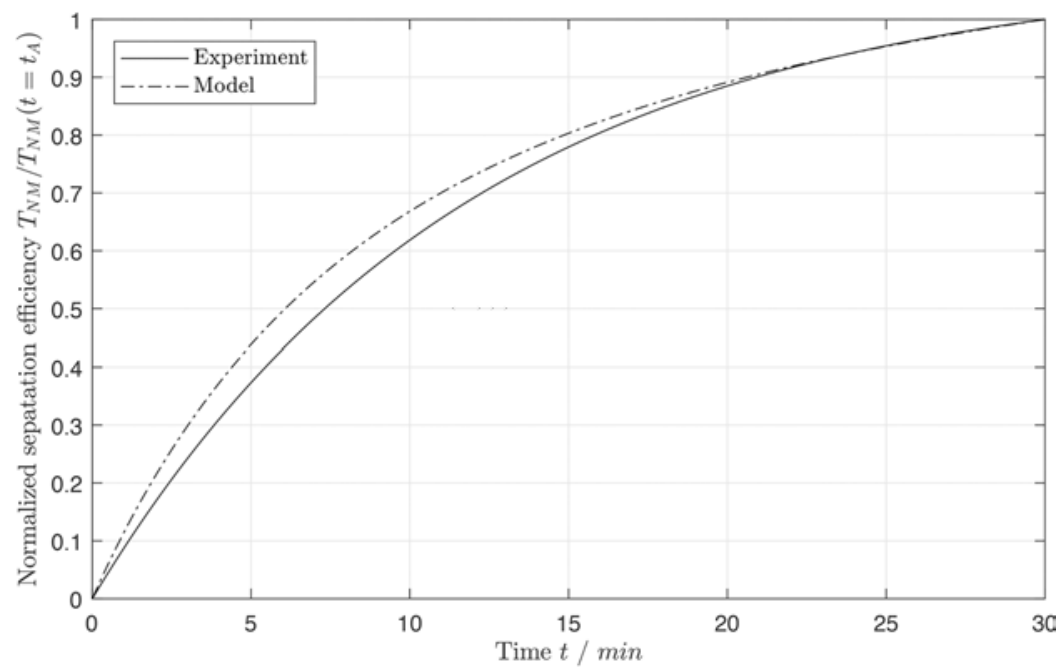

Fig. 8. Comparison between the normalized experimental and calculated agglomeration kinetic. Process parameters are $n=250 \mathrm{rpm}, c_{v, M}=0.01 \mathrm{vol} \%$, $\Phi_{v, \mathrm{NM}}=1$ and $I=10^{-3} \frac{\mathrm{mol}}{\mathrm{L}}$. 


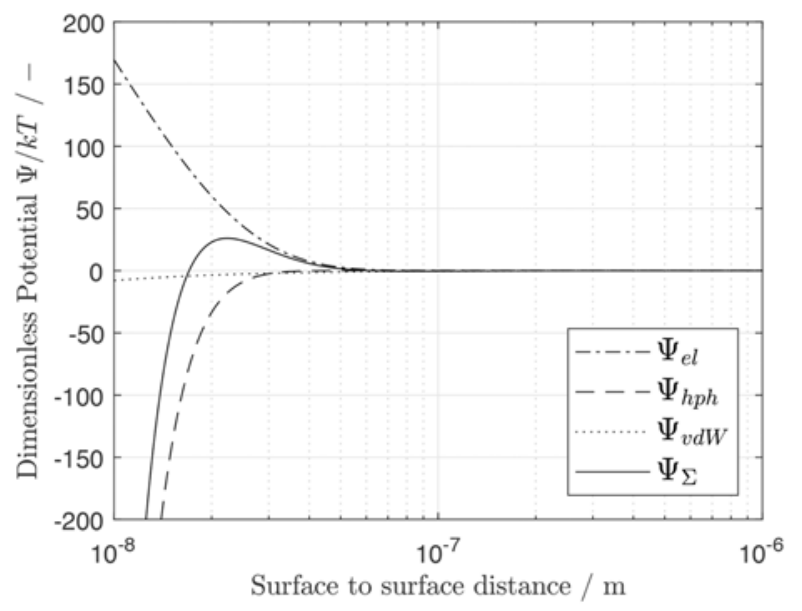

(a) $d_{1}=2 n m, d_{2}=4 n m$

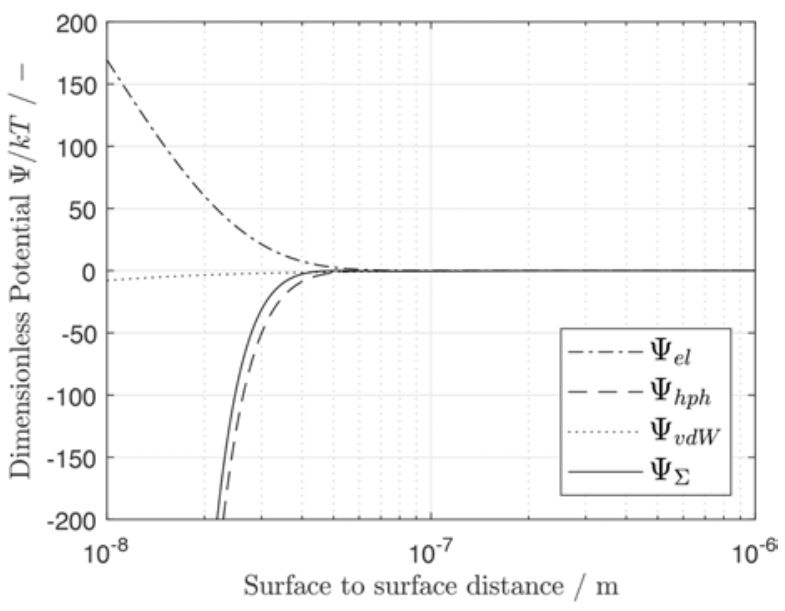

(b) $d_{1}=3 \mathrm{~nm}, d_{2}=6 \mathrm{~nm}$

Fig. 9. Interaction potentials between two PVC surfaces for critical model parameter values. Other relevant parameters are $I=10^{-3} \frac{\mathrm{mol}}{\mathrm{L}}, C_{1, \mathrm{NM}, \mathrm{NM}}=300 \frac{\mathrm{mN}}{\mathrm{m}}$, $C_{2, \mathrm{NM}, \mathrm{NM}}=10 \frac{\mathrm{mN}}{\mathrm{m}}$.

critical physical relationships and relative importance of the various agglomeration-relevant model parameters, although it may not provide final unambiguous values for the variables studied.

As mentioned above, by performing additional model calculations, various hard-to-measure results become accessible. The following paragraph aims to showcase the potential of these calculations and gives additional information about the investigated agglomeration problem. At first, the raw data is stored in the matrix $N(i, j, t)$ which describes the class- and time-dependent agglomerate number densities. A possible visualization of such 4-dimensional data is shown in Fig. 10 and is inspired by [7]. The concentration values on the z-axis are normalized to the sum of the initial number concentrations of the primary particles which allows for an easier estimate of the separation efficiency at any given time. The $y$-axis shows the number of primary particles per agglomerate $\left(N_{S}\right)$ and can thus be interpreted as agglomerate size. On the $x$-axis, the various possible agglomerate classes are distinguished while the gray values represent the fraction of magnetic particles (gray is magnetic, white is non-magnetic). Data illustration as in Fig. 10 produces a vivid understanding of the ongoing time-dependent processes and allows for a qualitative discussion. It shows a rapid decrease in magnetic particles while the agglomeration of non-magnetic primary particles is slower. However, for a further quantitative approach, the number-weighted particle size distributions as well as the according density distributions can be calculated. They are shown in Fig. 11. It becomes apparent that the fraction of non-magnetic primary particles represented by the left-most peak in the $q_{0}$ and the left-most increase in the $Q_{0}$ distribution is seemingly constant over the course of the agglomeration process. This can be quite misleading when conferring with Fig. 10 since a decrease in particle concentration is definitely present (otherwise the separation efficiency would be zero). However, since two smaller agglomerates combine to one larger agglomerate during the process, the absolute number of individual agglomerates decreases which makes it possible for the fraction of nonmagnetic primary particles to stay constant. On the other hand, it can be seen that the fraction of magnetic particles (second left-most peak in $q_{0}$ ) rapidly decreases. This illustrates the fact that the magnetic particles are destabilized due to the non-existence of a surface charge and agglomeration of them is only limited by collision. This effect can
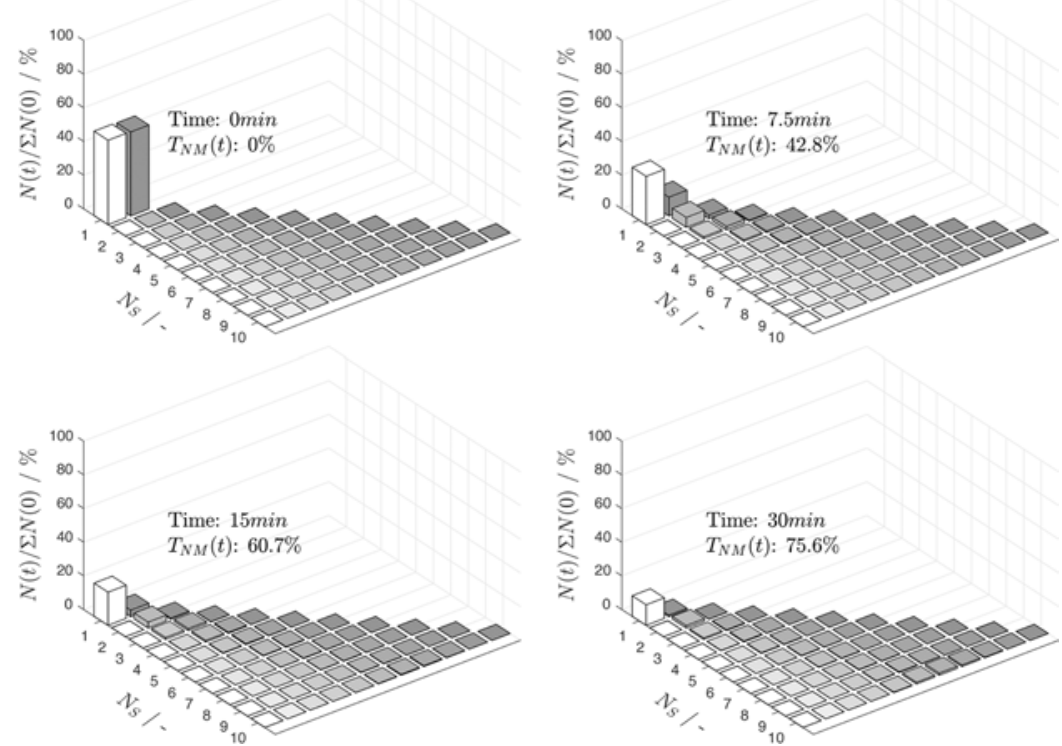

Fig. 10. Raw data of calculated results. Process parameters are $n=250 \mathrm{rpm}, c_{v, M}=0.01 \mathrm{vol} \%, t_{A}=30 \mathrm{~min}, \Phi_{v, \mathrm{NM}}=0.5 \mathrm{and} I=10^{-6} \frac{\mathrm{mol}}{\mathrm{L}}$. 

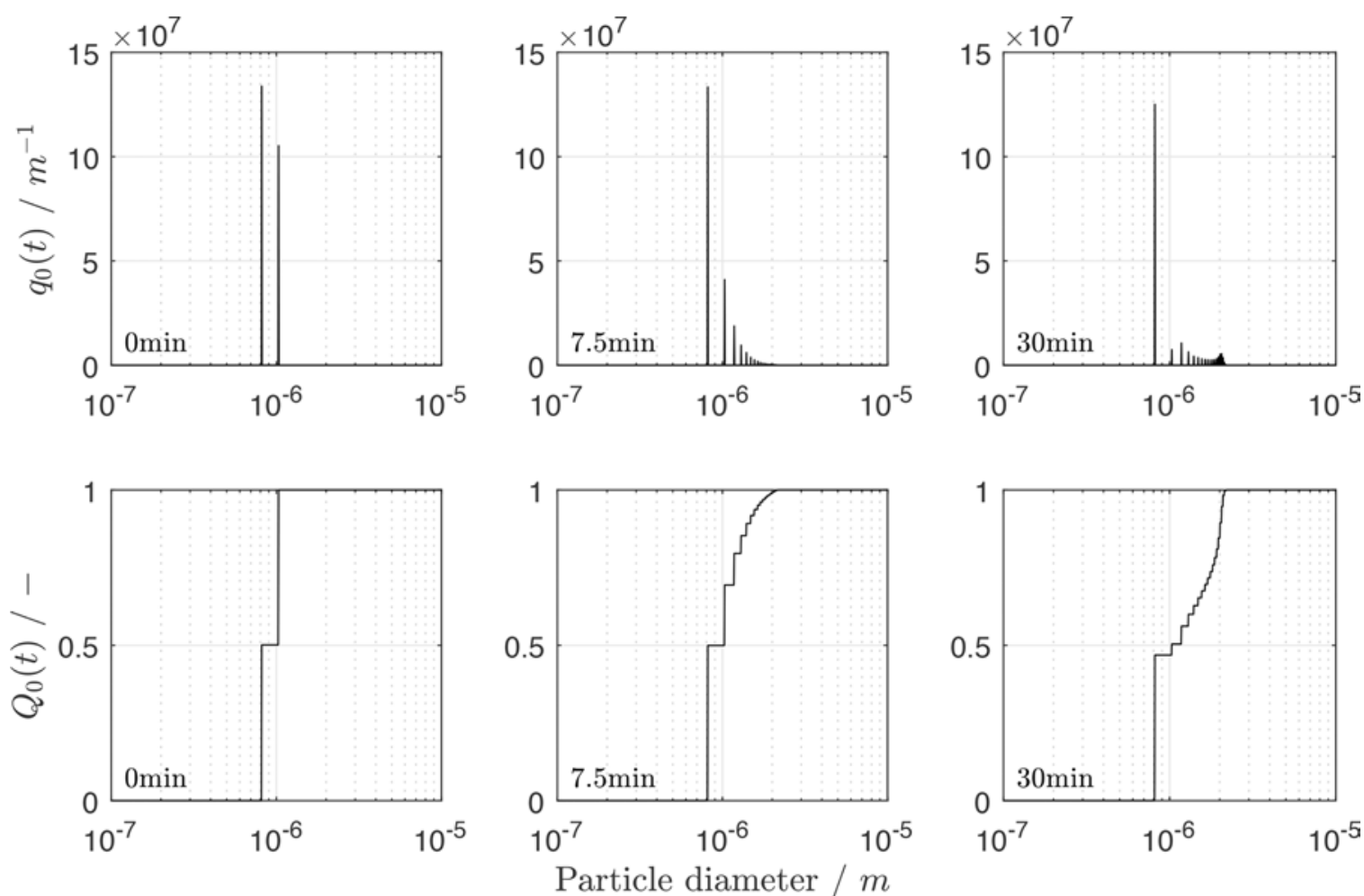

Fig. 11. Time-dependent number-weighted particle size $\left(Q_{0}\right)$ and particle size density distributions $\left(q_{0}\right)$.

further be seen when regarding the time-dependent agglomerate composition of a given agglomerate size, as shown in Fig. 12 for $N_{S}=10$. With increasing time, the mean composition shifts from $\approx 70 \%$ to $\approx 60 \%$ magnetic particles. The fast agglomeration of primary magnetic particles manifests itself in a large fraction of magnetic particles overall and especially after short agglomeration times. The shift of composition for increasing agglomeration times is due to the limitation of the system by $N_{S, \max }$ and the predefined concentration ratio $\Phi_{v, \mathrm{NM}}$. For an infinite time, the composition will converge to $\Phi_{v, \mathrm{NM}}$, however, Fig. 12 is able to describe the dynamic of this process.

\section{Conclusions}

Initially, an experimental approach for investigating hetero-agglomeration processes was used. Thereby, a DOE varying multiple process-relevant parameters was conducted, while the results showed a reasonable standard deviation and were in good accordance with the basic agglomeration theory. A further measurement of the agglomeration kinetics allowed for a deeper process characterization and basic simplifying mathematical expressions were found that were consistent with the data.

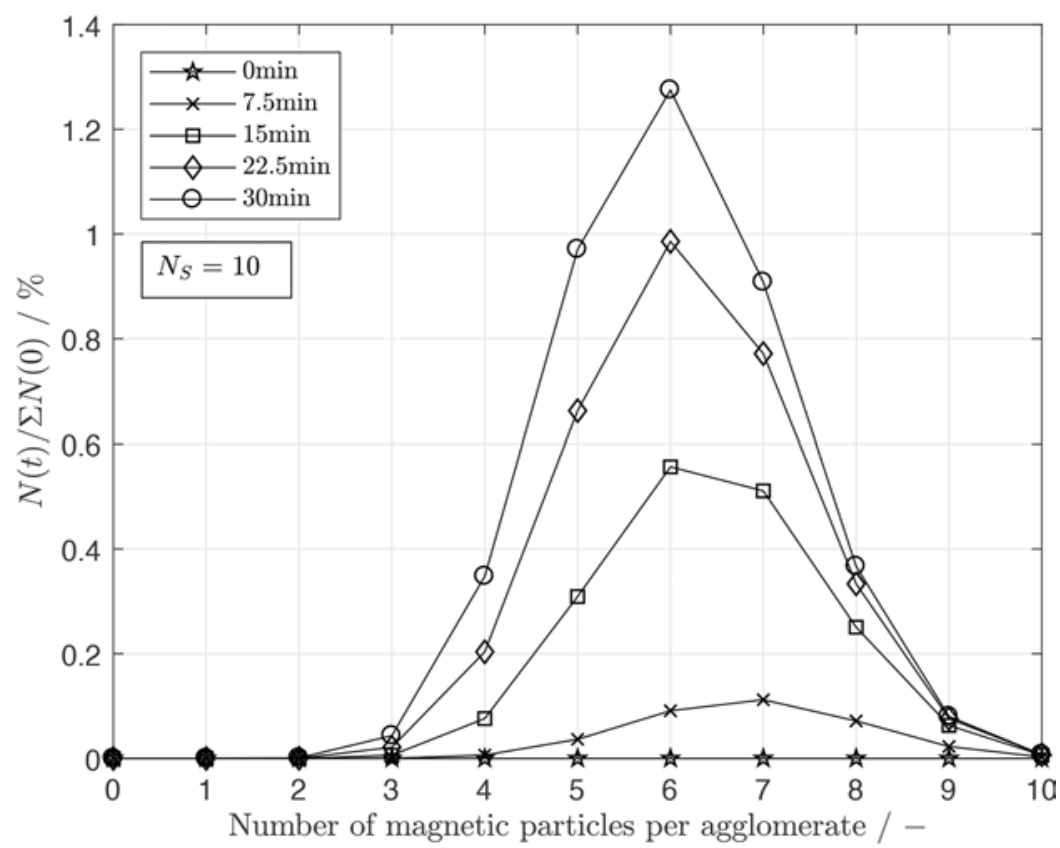

Fig. 12. Time-dependent agglomerate composition for $N_{S}=10$. 
However, since the experimental data cannot access valuable information as for example time-dependent agglomerate compositions, a population balance equation was used to gain a different angle of investigation on the problem. Here, a new and simple approach for estimating material data of hetero-agglomerates was presented and implemented in the calculations. From a physical standpoint, regarding different collision cases and using the primary particle properties seems more justifiable than e.g. volume-weighted averaging in a surfacecontrolled process.

Due to the used material systems, the hydrophobic interactions had to be included in the population balance equations. However, the necessary material properties are hard to measure and thus an estimate of this interaction potential is delicate. This work strived in a new direction of gaining information about these parameters by comparing calculated and experimental results. The so found model parameters could then be used to re-calculate the measured DOE and the comparison of resulting regression models showed that the model calculations are able to describe the fundamental physical processes. In addition to that, the comparison of theoretically and experimentally gained agglomeration kinetics also emphasizes the basic soundness of the calculations. Hence, the presented model could be validated by multiple experimental means.

Finally, this work showcases the additional information gain though model calculation and the possibilities for a better process understanding arising from them. Calculated data can be visualized in various manners and examples are given for potential qualitative and quantitative interpretations.

\section{Acknowledgment}

The authors acknowledge the support in contact angle measurement probe preparation by the Institute for Technical Chemistry and Polymer Chemistry. The research project is funded by the DFG (German Research Foundation) in the priority program 2045 Highly specific and multidimensional fractionation of fine particle systems with technical relevance.

\section{References}

[1] T.Y. Ying, S. Yiacoumi, C. Tsouris, High-gradient magnetically seeded filtration, Chem. Eng. Sci. 55 (6) (2000) 1101-1113, https://doi.org/10.1016/S00092509(99)00383-8.

[2] E. Förster, A. Schmidt, F. Rhein, H. Anlauf, H. Nirschl, Hetero agglomeration processes in a high-gradient magnetic filter design, Chem. Eng. Technol. 39 (3) (2016)
477-483, https://doi.org/10.1002/ceat.201500343.

[3] A.D. Ebner, J.A. Ritter, H.J. Ploehn, Magnetic hetero-flocculation of paramagnetic colloidal particles, J. Colloid Interface Sci. 225 (1) (2000) 39-46, https://doi.org/ 10.1006/jcis.2000.6739.

[4] D. Ramkrishna, Population Balances: Theory and Applications to Particulate Systems in Engineering, Academic Press, San Diego, CA, 2000, https://doi.org/10. 1016/B978-0-12-576970-9.X5000-0.

[5] D. Ramkrishna, M.R. Singh, Population balance modeling: current status and future prospects, Annu. Rev. Chem. Biomol. Eng. 5 (1) (2014) 123-146, https://doi.org/ 10.1146/annurev-chembioeng-060713-040241.

[6] P. Taboada-Serrano, C.-J. Chin, S. Yiacoumi, C. Tsouris, Modeling aggregation of colloidal particles, Curr. Opin. Colloid Interface Sci. 10 (3) (2005) 123-132, https://doi.org/10.1016/j.cocis.2005.07.003.

[7] C. Tsouris, S. Yiacoumi, T.C. Scott, Kinetics of heterogeneous magnetic flocculation using a bivariate population-balance equation, Chem. Eng. Commun. 137 (1) (1995) 147-159, https://doi.org/10.1080/00986449508936373.

[8] C.-J. Chin, S. Yiacoumi, C. Tsouris, Shear-induced flocculation of colloidal particles in stirred tanks, J. Colloid Interface Sci. 206 (2) (1998) 532-545, https://doi.org/ 10.1006/jcis.1998.5737.

[9] S. Chimmili, D. Doraiswamy, R.K. Gupta, Shear-induced agglomeration of particulate suspensions, Ind. Eng. Chem. Res. 37 (6) (1998) 2073-2077, https://doi.org/ 10.1021/ie9706368.

[10] V. Oles, Shear-induced aggregation and breakup of polystyrene latex particles, J. Colloid Interface Sci. 154 (2) (1992) 351-358, https://doi.org/10.1016/00219797(92)90149-G

[11] J. Skvarla, F. Zelenak, Magnetic-hydrophobic coagulation of paramagnetic minerals: a correlation of theory with experiments, Int. J. Miner. Process. 68 (1-4) (2003) 17-36, https://doi.org/10.1016/S0301-7516(02)00042-X.

[12] H.K. Christenson, P.M. Claesson, Direct measurements of the force between hydrophobic surfaces in water, Adv. Colloid Interface Sci. 91 (3) (2001) 391-436, https://doi.org/10.1016/S0001-8686(00)00036-1.

[13] J. Israelachvili, R. Pashley, The hydrophobic interaction is long range, decaying exponentially with distance, Nature 300 (1982) 341, https://doi.org/10.1038/ $300341 \mathrm{a} 0$.

[14] J.N. Israelachvili, Intermolecular and Surface Forces, 3rd ed., Elsevier, Amsterdam, Heidelberg [U.A.], 2011, https://doi.org/10.1016/C2009-0-21560-1.

[15] K. Menzel, C.W. Windt, J.A. Lindner, A. Michel, H. Nirschl, Dipolar openable halbach magnet design for high-gradient magnetic filtration, Sep. Purif. Technol. 105 (Supplement C) (2013) 114-120, https://doi.org/10.1016/j.seppur.2012.12.019.

[16] J. Svoboda, Magnetic Techniques for the Treatment of Materials, Springer Netherlands, 2004, https://doi.org/10.1007/1-4020-2107-0.

[17] P.D. Berger, Experimental Design: With Applications in Management, Engineering and the Sciences, 2nd ed., Springer, Cham, SpringerLink: Bücher, 2018, https://doi. org/10.1007/978-3-319-64583-4.

[18] N. Fuchs, Über die Stabilität und Aufladung der Aerosole, Zeitschrift für Physik 89 (11) (1934) 736-743, https://doi.org/10.1007/BF01341386.

[19] E.P. Honig, G.J. Roebersen, P.H. Wiersema, Effect of hydrodynamic interaction on the coagulation rate of hydrophobic colloids, J. Colloid Interface Sci. 36 (1) (1971) 97-109, https://doi.org/10.1016/0021-9797(71)90245-1.

[20] B. Derjaguin, L. Landau, Theory of the stability of strongly charged lyophobic sols and of the adhesion of strongly charged particles in solutions of electrolytes, Prog. Surf. Sci. 43 (1) (1941) 30-59, https://doi.org/10.1016/0079-6816(93)90013-L.

[21] M. Elimelech, Particle Deposition and Aggregation: Measurement, Modelling and Simulation 1st Edition, Colloid and Surface Engineering Series, ButterworthHeinemann, Woburn, Mass, 1998, https://doi.org/10.1016/B978-0-7506-7024-1. X5000-6. 
Karlsruher Institut für Technologie

\section{Repository KITopen}

Dies ist ein Postprint/begutachtetes Manuskript.

Empfohlene Zitierung:

Rhein, F.; Ruß, F.; Nirschl, H.

Collision case model for population balance equations in agglomerating heterogeneous

colloidal systems: Theory and experiment.

2019. Colloids and surfaces / A, 572.

doi: $10.5445 / \mathrm{IR} / 1000094675$

Zitierung der Originalveröffentlichung:

Rhein, F.; Ruß, F.; Nirschl, H.

Collision case model for population balance equations in agglomerating heterogeneous colloidal systems: Theory and experiment.

2019. Colloids and surfaces / A, 572, 67-78.

doi: $\underline{10.1016 / j . c o l s u r f a .2019 .03 .089}$ 